Bundesgesundheitsbl - Gesundheitsforsch Gesundheitsschutz 2009 · 52:238 DOI 10.1007/s00103-009-0785-9 Online publiziert: 31. Januar 2009 ๑) Springer Medizin Verlag 2009

\title{
Lebensphasenspezifische Gesundheit von Kindern und Jugendlichen in Deutschland
}

\author{
Ergebnisse des Nationalen Kinder- und \\ Jugendgesundheitssurveys
}

ter von o-17 Jahren mittels standardisierter Methoden im gesamten Bundesgebiet erfasst. Mit KiGGS konnten Informationslücken zu wesentlichen Bereichen der Kinder- und Jugendgesundheit geschlossen werden, wie z.B. zur körperlichen und seelischen Gesundheit, zu gesundheitlich bedeutsamen Verhaltensweisen sowie zur Inanspruchnahme medizinischer Versorgungsangebote. Bei der hier vorliegenden Ergebnisdarstellung wurde berücksichtigt, dass Kindheit und Jugend ein sehr differenziert zu betrachtender Altersbereich im Hinblick auf physiologische, psychologische und soziologische Entwicklungsprozesse sind. Entsprechend der Alters- und Entwicklungsspezifik der o- bis 17-Jährigen wird in dem Bericht eine le- bensphasenspezifische Betrachtung und Aufbereitung der KiGGS-Ergebnisse vorgenommen. Dabei werden die Lebensphasen frühes Kindesalter, Schul- und Jugendalter betrachtet und es erfolgt eine Differenzierung der Indikatoren nach dem sozioökonomischen Status der Kinder und Jugendlichen, ihrem Migrationshintergrund sowie nach der Wohnregion. Die lebensphasenbezogene Betrachtungsweise unter Berücksichtigung sozialer Gegebenheiten leistet einen Beitrag zu einer kontinuierlichen und problemorientierten nationalen Gesundheitsberichterstattung zu Kindern und Jugendlichen und liefert Ansatzpunkte für präventiv orientierte Maßnahmen. 